\title{
Analysis and Validation of Putative Substances Involved in Fatal Poisonings
}

\author{
Christopher K. Hansen • John Kashani • Bruce Ruck • \\ Steven Marcus
}

Published online: 8 December 2011

(C) American College of Medical Toxicology 2011

\begin{abstract}
Each year, poison control centers throughout the United States respond to over 4 million calls for help in treating individuals exposed to toxic substances. Although most cases develop no or minimal clinical effects, a small proportion of patients who receive medical care for overdoses with poison center consultation expire. When such cases are investigated by a medical examiner, the postmortem toxicology results may show substances other than those considered in the consultation with the poison center. We sought to determine the characteristics of discordance in fatal cases between the toxic substances reported to a regional poison control center and postmortem toxicology results. We conducted a retrospective study of the New Jersey regional poison control center records of all fatal cases between the years 1986 and 2006. Substances reported as putative agents to the poison center were compared to the postmortem toxicology results obtained by the medical examiner. The frequencies and characteristics surrounding discordance were examined. Of the 708 fatal cases reported to our poison center within the study
\end{abstract}

Funding The authors did not receive funding of any kind for this study.

Data Presentation Preliminary data presented at the 2008 North American Congress of Clinical Toxicology, Toronto, Canada.

C. K. Hansen · J. Kashani • B. Ruck · S. Marcus

New Jersey Poison Information and Education System, Department of Preventive Medicine and Community Health, UMDNJ-New Jersey Medical School,

Newark, NJ, USA

S. Marcus $(\bowtie)$

New Jersey Poison Information and Education System, 140 Bergen Street G1600,

Newark, NJ 07101-1709, USA

e-mail: smarcus@njpies.org period, complete postmortem toxicological evaluations were available for 206 (29.0\%). Comparison of putative agents between information obtained by history and at postmortem evaluation showed discordance in 41 (19.9\%). In a substantial number of fatal cases receiving poison center consultation, substances were found at the time of postmortem examination that were not considered in the poison center consultation. The reasons for this discordance may include a lack of thorough history-taking or a cognitive bias to the substances initially reported.

Keywords Fatal poisoning - Poison control center . Postmortem

\section{Introduction}

Poisoning and drug overdose both intentional and unintentional are leading causes of morbidity and mortality in the United States [1]. In 2007, there were 40,059 intentional and unintentional poisoning deaths reported in the United States [2]. The economic costs of poisoning are approximately $\$ 26$ billion in medical expenses annually, making up $6 \%$ of the economic costs of all injuries in the United States [3]. Nationally, regional poison control centers (RPCCs) respond to over 4 million calls for help in treating individuals thought to be exposed to toxic substances. Although the vast majority of such calls are determined to be non life-threatening and are treated outside of a health care facility, some cases are deemed to be potentially severe enough to warrant emergent management in a hospital. For these patients, RPCCs play an important role by providing telephone toxicology consultation to the treating medical professionals [4]. Despite this consultation, a proportion of patients expire. 
In most of the United States, accidental deaths are reportable illnesses and are often investigated with postmortem examinations. If a postmortem is performed, the investigation routinely includes an autopsy and toxicologic testing of blood and other tissue specimens for a core panel of substances as well as others chosen at the discretion of the medical examiner. It was our experience that, in a substantial number of patients who received RPCC consultation and later expired, the postmortem toxicology results did not correspond to the data collected during the initial call to the RPCC.

To our knowledge, no previous study has specifically examined discrepancies in the medications or substances reported to the poison center and those found postmortem. Our main objective was to identify and analyze such discrepancies and to describe the frequencies and characteristics surrounding any discordance.

\section{Methods}

We conducted a retrospective analysis of deceased individuals whose healthcare provider contacted the New Jersey Poison Information and Education System (NJPIES) for consultation following a drug overdose or toxic exposure between 1986 and 2006. NJPIES is the only RPCC for the entire state of New Jersey (population 8,707,739) and operates a toll-free telephone hotline that provides consultation to the lay public and healthcare providers on the medical management of poisonings and toxic exposures. Every call to the center generates a medical record that is stored in a centralized computer database where patient demographics and data pertinent to the presentation and management of the patient are recorded. All cases are managed by specialists in poison information, credentialed as either physicians, nurses, or clinical pharmacists with oversight provided by medical toxicologists.

If a patient is determined to have expired, the hospital record and results of the postmortem examination are requested by the RPCC's medical director. New Jersey has a medical examiner system that encompasses three regional medical examiner offices covering seven of the 21 counties in the state. The remaining 14 counties each operate their own medical examiner office for deaths that occur within their respective county. All autopsies were conducted by a board certified forensic pathologist. The seven counties in the regional medical examiner system utilize a centralized state toxicology laboratory for forensic toxicological analysis. This laboratory is run by a board certified forensic toxicologist. The remaining medical examiner offices utilize a private laboratory that tests for an identical core panel of substances. The basic panel did not change drastically over the study period and is listed in Table 1 .
Any substances added to the original 1986 core panel are noted. Additionally, if there was any suspicion by the medical examiner of a substance not found in the core panel such as an insecticide or detergent, this could be specially added to the toxicological analysis.

We analyzed all deaths between Janurary 1, 1986 and December 31, 2006. Demographic information on callers including the year, age, sex, location of the call by county, medical qualifications of the caller (i.e. physician, nurse, paramedic, etc.), the substances believed to have been taken or exposed to and the estimated amount of the exposure, and any follow-up advice given by the poison specialist over the phone were extracted and entered into the dataset. These were mandatory data fields completed by the Specialist in Poison Information (SPI). If a patient's substance list was updated by follow-up calls to the RPCC during their treatment, these substances were included in the dataset up until the time of death. Postmortem toxicology results were also extracted for these cases and entered into the dataset. Cases were included in the study only if they had a complete RPCC call record and had an autopsy performed that included a postmortem toxicological analysis of blood, bile, urine, and brain matter. Cases were excluded if they did not have an autopsy performed, had only an external examination performed, or were missing a postmortem toxicological analysis from their autopsy record. All deaths occurred in either the ED or inpatient unit of a hospital facility. This study did not examine cases where death occurred in the prehospital setting.

The result of the toxicological evaluation was compared with the substances reported to the RPCC as extracted from the RPCC case records. We only included toxicology studies performed on blood or other tissues obtained after death. For descriptive purposes, we categorized toxic substances into the following three groups: opioid analgesics and sedatives with abuse potential, other prescription medications that do not fall in the above group, and nonprescription medications and other toxic substances. These categories were not mutually exclusive and cases in which substances fell into more than one category were placed in a fourth category: multiple drugs or toxic substances identified. Substances that were therapeutically administered during the patient's hospital course such as morphine, diazepam, or phenytoin were excluded from the dataset if they were documented for therapeutic use in the patient's record.

Cases for which the substances reported to the RPCC were not found in the postmortem toxicological analysis were classified as discordant. If the substances were not known when the RPCC was contacted but were subsequently discovered on postmortem toxicology, the case was classified as unknown substance. If the substances initially reported were not known but were later identified during the course of treatment up until the time of death, the case 
Table 1 Core panel of substances tested

1986 panel of substances $(n=98)$

\begin{tabular}{|c|c|c|c|c|}
\hline Volatiles: & cannaboids & doxepin & methamphetamine & promethazine \\
\hline acetone & carbamazepine & doxylamine & methapyriline & propoxyphene \\
\hline ethanol & chloral hydrate & flurazepam & methaqualone & protriptyline \\
\hline isopropanol & chlordiazepoxide & glutethimide & methphenytoin & pyrilamine \\
\hline methanol & chlorpheniramine & hydromorphone & morphine (free) & quinidine \\
\hline ethylene glycol & chlorpromazine & haloperidol & nitrazepam & quinine \\
\hline Drugs and Metabolites: & clonazepam & hydrocodone & nordiazepam & salicylates \\
\hline 6-mono-acetyl morphine & cocaethylene & imipramine & norpropoxyphene & scopolamine \\
\hline acetaminophen & cocaine & ketamine & nortriptyline & secobarbital \\
\hline alprazolam & codeine & lidocaine & oxazepam & talbutal \\
\hline amitriptyline & cyanide & lorazepam & papaverine & temazepam \\
\hline amobarbital & cyproheptadine & loxapine & pentazocine & thiopental \\
\hline amoxapine & demoxepam & maprotiline & pentobarbital & thioridazine \\
\hline amphetamine & desalkylflurazepam & MDA & phencyclidine & tranylcypromine \\
\hline barbital & desipramine & MDMA & phenmetrazine & trazodone \\
\hline benztropine & dextromethorphan & meperidine & phenobarbital & triazolam \\
\hline benzoylecgonine & diazepam & mephobarbital & phenylpropanolamine & trihexyphenidyl \\
\hline brompheniramine & diphenhydramine & meprobamate & phenytoin & trimipramine \\
\hline butabarbital & diphenoxylate & mesoridazine & procainamide & tripelennamine \\
\hline butalbital & disopyramide & methadone & procyclidine & \\
\hline
\end{tabular}

The following substances were added in 1996: bupivacaine, carisoprodol, chlorpropamide, cyclobenzaprine, diltiazem, ephedrine, estazolam, ethosuximide, fenfluramine, fluoxetine, hydroxyzine, levorphanol, methsuximide, metoprolol, n-acetyl procainamide, oxycodone, paroxitine, phendimetrazine, phenethylamine, phensuximide, phentermine, prazepam, primidone, propranolol, pseudoephedrine, sertraline, theophylline, venlafaxine, and verapamil. Ethchlorvynol was removed from the panel

The following substances were added in 2006: bupropion, buspirone, chlorzoxazone, citolapram, colchicine, fentanyl, ibuprofen, mirtazepine, naproxen, nefazodone, olanzepine, oxcarbazepine, propofol, quetiapine, tramadol, warfarin, and zolpidem

was classified as either discordant or concordant depending upon the results of the postmortem toxicology. All other cases for which the substances reported were present postmortem were classified as concordant.

All data was entered directly into a Microsoft Excel spreadsheet. Data analysis was performed using SPSS (IBM, Somers, NY). Means were analyzed using a oneway analysis of variance. The University of Medicine and Dentistry of New Jersey Institutional Review Board approved this study.

\section{Results}

Between the years 1986 and 2006, there were 708 cases of toxic exposures to the RPCC that resulted in fatality. Of these, 502 were excluded due to incomplete information with 206 (29.0\%) fitting the inclusion criteria previously described. The mean age for all cases was $37.8 \pm 17.6$ years (range 3 days89 years). One hundred and twenty-two (59.0\%) were male. The substances most frequently involved were nonprescription products and other substances $(35.0 \%)$ followed by multiple drugs or toxic substances $(26.2 \%)$. Prescription medications accounted for $21.8 \%$ of cases and $17 \%$ of cases involved opioid analgesics and sedatives with abuse potential. The types of substances involved varied by age group (Fig. 1). The 0 19-year-old age group comprised $12.1 \%$ of the study population. The most frequent substances involved in this age group were nonprescription products and other toxic substances (52\% of these cases). The 30-39-year-old age group had the highest proportion of cases in our study with $28.6 \%$ of all cases. Nonprescription products and other substances were involved in $39.0 \%$ of cases in this age group.

The level of medical training of the initial caller to the poison center included 144 (69.6\%) physicians, 52 (25.2\%) nurses, $5(2.4 \%)$ other healthcare providers, and $5(2.4 \%)$ nonhealthcare providers. The callers classified as other healthcare providers included $1(0.5 \%)$ physician assistant, 2 (1\%) emergency medical technicians, and $2(1 \%)$ paramedics.

Our analysis of substances reported to the RPCC and those found upon postmortem toxicology testing by the medical examiner showed $41(19.9 \%)$ cases that demonstrated discordance. In 17 of these cases, the substances reported to have been taken did not correspond to the 
Fig. 1 Types of substances involved in fatal poisonings by age group

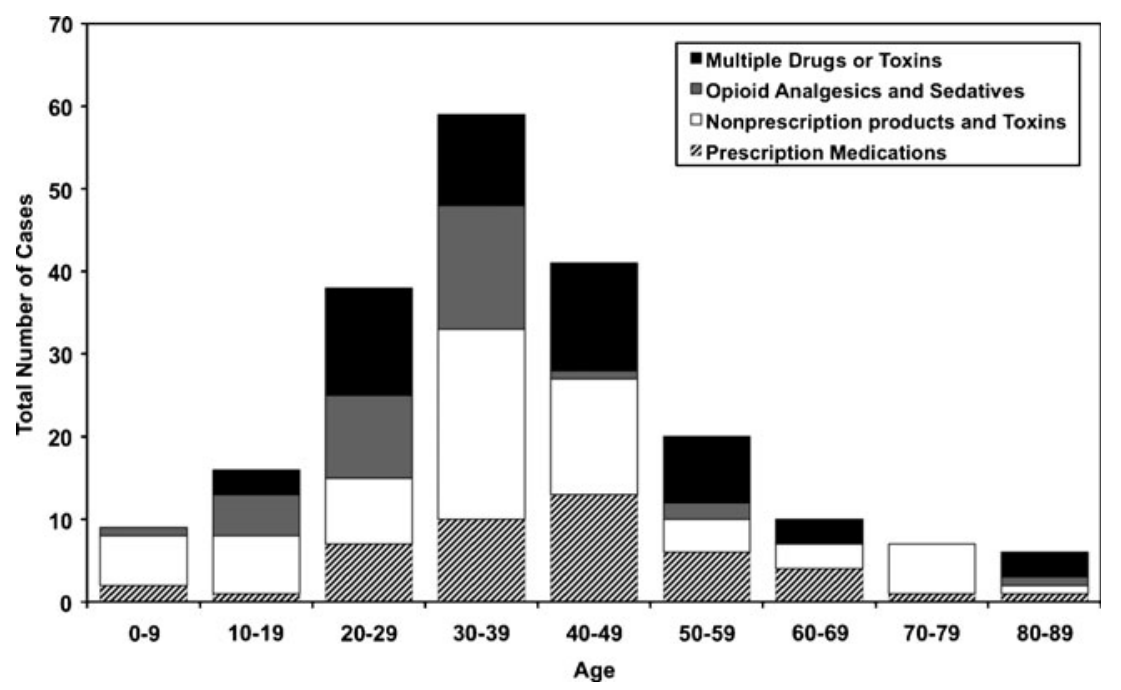

substances found in the postmortem toxicology analysis (Table 2). In one case, an organophosphate was reported that did not correspond to the dinitrophenol that was discovered in the postmortem toxicology. This was discovered only after additional testing beyond the core panel. In the other 24 cases, no substances were found postmortem (Table 3). For cases in which uncommon substances were reported such as bifenthrin, piroxicam, kelthane, orthene, detergents, and pesticides, specific testing did not reveal these substances. In five cases $(2.4 \%)$, the substances involved were unable to be identified during the initial call or subsequent follow up with the RPCC but were revealed on postmortem toxicology (Table 4). Among the concordant cases, the mean age was $36.8 \pm 17.9$ years (range 3 days -89 years) and among discordant cases, the mean age was $41.7 \pm 17.0$ years (range 4 months -81 years). Among the cases where the substances reported the RPCC were unknown, the mean age was $38.2 \pm 9.0$ years (range 2548 years) (Table 5 ).

Of the discordant cases, $53.7 \%$ were male and $61.5 \%$ of the concordant cases were male. Forty percent of the unknown substance cases were male. The toxin type most frequently encountered in discordant cases was over-thecounter drugs and other toxic substances, including carbon
Table 2 Substances believed to have been taken compared to substances found postmortem when there was discordance $(n=17)$

${ }^{\mathrm{a}}$ Indicates a substance was reported to the RPCC and found postmortem. Substances found postmortem were identified in blood or other tissues obtained after death

\begin{tabular}{|c|c|c|}
\hline Substances initially reported to RPCC & Substances found postmortem & Year \\
\hline Beta-adrenergic antagonist & Fluoxetine, hydrocodone & 2005 \\
\hline $\begin{array}{l}\text { Acetaminophen, bupropion, clonazepam, } \\
\text { olanzapine, oxcarbazepine }\end{array}$ & $\begin{array}{l}\text { Diazepam, nordiazepam, oxazepam, } \\
\text { propoxyphene, norpropoxyphene }\end{array}$ & 2004 \\
\hline Citalopram, diltiazem & Benzodiazepines & 2002 \\
\hline $\begin{array}{l}\text { Amitriptyline }{ }^{\mathrm{a}} \text {, aspirin }{ }^{\mathrm{a}} \text {, chlordiazepoxide, } \\
\text { triazolam, paroxetine, clonazepam }\end{array}$ & $\begin{array}{l}\text { Amitriptyline }{ }^{\mathrm{a}} \text {, salicylate }{ }^{\mathrm{a}} \text {, nortriptyline, } \\
\text { nordiazepam }\end{array}$ & 1999 \\
\hline Unknown organophosphate & Dinitrophenol & 1999 \\
\hline Nifedipine & Benzodiazapine, morphine, opiates & 1999 \\
\hline Raid $^{\circledR}$ roach spray & Cocaine & 1997 \\
\hline Theophylline $^{\mathrm{a}}$ & Theophylline ${ }^{\mathrm{a}}$, clonazepam, doxepin, nortriptyline & 1995 \\
\hline Copper dust & Carbon monoxide & 1992 \\
\hline Methanol, nortriptyline & Ethylene glycol & 1991 \\
\hline $\begin{array}{l}\text { Codeine }^{\mathrm{a}} \text {, acetaminophen, ethanol, } \\
\text { propoxyphene napsylate }\end{array}$ & Codeine $^{\mathrm{a}}$, glutethimide, morphine & 1990 \\
\hline Red Cross toothache ${ }^{\circledR}(85 \%$ Eugenol) & Amitriptyline, codeine, ethanol, nortriptyline & 1989 \\
\hline $\begin{array}{l}\text { Acyclovir, cocaine, lorazepam, mesoridazine, } \\
\text { phenelzine }\end{array}$ & $\begin{array}{l}\text { Chlorpheniramine, dextromethorphan, } \\
\text { pseudoephedrine }\end{array}$ & 1989 \\
\hline Ethanol $^{\mathrm{a}}$ & $\begin{array}{l}\text { Ethanol }{ }^{\mathrm{a}} \text {, chlordiazepoxide, demoxepam, } \\
\text { doxepin }\end{array}$ & 1989 \\
\hline Methadone, phenteramine & Benzoylecgonine, cocaine & 1989 \\
\hline Lysergic acid diethylamide, phencyclidine & Benzoylecgonine, cocaine & 1988 \\
\hline Aspirin, butalbital, caffeine, diazepam & Ethylene glycol & 1986 \\
\hline
\end{tabular}


Table 3 Substances reported to the RPCC for which no substances were detected in postmortem toxicology $(n=24)$

\begin{tabular}{ll}
\hline Substances & Year \\
\hline Fenfluramine, phentermine & 2006 \\
Acetaminophen, hydrocodone & 2005 \\
Bifenthrin & 2005 \\
Amlodipine, benzodiazepine, doxepin, valsartan & 2004 \\
Amlodipine, clonidine, cocaine, ethanol, lansoprazole, & 2003 \\
metformin & 2002 \\
Piroxicam & 2002 \\
Acetaminophen, ethanol, nifedipine & 2001 \\
Haloperidol, lorazepam, risperidone, St. John's wort & 2001 \\
Botulism toxin & 2000 \\
Amitriptyline, heroin & 2000 \\
Unknown drug (2 cases) & 1999 \\
& 1999 \\
Atenolol, diazepam, fluoxetine, simvastatin & 1998 \\
Doxepin, lorazepam, olanzapine, trazedone, venlafaxine & 1997 \\
Kelthane, orthene & 1997 \\
Doxepin & 1995 \\
Phenytoin & 1995 \\
Albuterol, cephalexin, theophylline & 1992 \\
Laundary detergent & 1992 \\
Ethanol, ethylene glycol (2 cases) & 1989 \\
D-con fogger (insecticide) & 1990 \\
Verapamil & 1990 \\
Cocaine & 1988 \\
\hline
\end{tabular}

monoxide, with 15 (32.6\%) cases. Multiple drugs or toxic substances were identified in $11(26.8 \%)$ cases showing discordance and in $41(25.6 \%)$ cases showing concordance. Finally, opioid analgesics and sedatives with abuse potential and other prescription drugs were identified in 9 $(22.0 \%)$ and $6(14.6 \%)$ cases showing discordance, respectively.

We analyzed the time elapsed from the original call to the RPCC until the patient was pronounced dead. Of all the

Table 4 Substances found postmortem when an unknown drug was initially reported $(n=5)$

\begin{tabular}{ll}
\hline $\begin{array}{l}\text { Substances initially } \\
\text { reported to RPCC }\end{array}$ & Substances found postmortem \\
\hline Unknown drug & Phenytoin, acetone \\
& Amitriptyline, nortriptyline \\
& Nortriptyline, codeine, \\
oxycodone, desmethylsertraline, phenytoin \\
Benzoylecgonine, ecgonine methyl \\
ester, morphine \\
Cocaine, benzoylecgonine
\end{tabular}

cases included in the study, the mean elapsed time until death was 25.5 hours ( $\mathrm{SD} \pm 64.3 \mathrm{~h}$ ). The longest time until death was 25.04 days and the shortest time was $0 \mathrm{~h}$ in two cases. These two patients were pronounced dead as the toxic substances were simultaneously being reported to the RPCC. The mean elapsed time until death was $25.4 \mathrm{~h}$ (SD \pm $67.1 \mathrm{~h})$ in the concordant cases, $27.6 \mathrm{~h}(\mathrm{SD} \pm 57.3 \mathrm{~h})$ in the discordant cases, and $14.5 \mathrm{~h}(\mathrm{SD} \pm 18.2 \mathrm{~h})$ in the unknown substances reported group. A one-way analysis of variance showed that mean time until death was not statistically significant among the discordant, concordant, and unknown substance groups, $F(2,203)=.01, p=0.91$.

There were no significant trends over time noted in either the total number of cases or the number of discordant or unknown cases over the course of the study period. Over 3 -year intervals, the mean numbers of total $(F(6,14)=1.23$, $p=0.35)$, discordant $(F(6,14)=0.53, p=0.77)$, and unknown cases $(F(6,14)=0.33, p=0.9)$ in our study were not significantly different.

\section{Discussion}

Over the 20-year study period, the RPCC encountered an average of 35 cases per year that resulted in fatality. Our results demonstrate that a significant amount of fatal cases in which the RPCC was utilized for treatment consultation showed discordance between the initial substances reported and the postmortem toxicology results.

Among the types of substances involved in poison related deaths, the most discordant was seen in nonprescription and other toxic substances with $32.6 \%$ of cases. This is likely due to the inherent difficulty present in diagnosing the toxic effects of nonpharmaceutical substances such as industrial chemicals. In addition, over-the-counter products and herbal products are often not considered as "medications" or are considered safe by the nature of being considered over-the-counter. Further, the postmortem toxicological evaluation for OTC substances is limited. Careful attention to history of exposure to these substances is often forgotten.

Several studies have described cognitive biases that may affect clinical decision-making. The phenomenon known as anchoring may occur during history-taking in the critically ill patient and cause the clinician to be unduly persuaded by salient features in the initial presentation $[5,6]$. Thus, the healthcare provider might anchor to the patients' symptoms early in the presentation and fail to consider alternative diagnoses. In several of the cases in which there was discordance, the substances reported to the RPCC have vastly different clinical presentations than the substances that were identified postmortem. In such cases, an overreliance on history may lead to therapeutic error, which can adversely affect clinical outcomes. 
Table 5 Characteristics of concordant and discordant cases 1986-2006

\begin{tabular}{|c|c|c|c|c|}
\hline Case variables & Concordant $(n=160)$ & Discordant $(n=41)$ & Unknown $(n=5)$ & All cases $(n=206)$ \\
\hline Age, years, mean $\pm \mathrm{SD}$ & $36.8 \pm 17.9$ & $41.7 \pm 17.0$ & $38.2 \pm 9.0$ & $37.8 \pm 17.6$ \\
\hline \multicolumn{5}{|l|}{ Sex, no. $(\%)$} \\
\hline Male & $98(61.3)$ & $22(53.7)$ & $2(40.0)$ & $122(59.2)$ \\
\hline Female & $62(38.8)$ & $19(46.3)$ & $3(60.0)$ & $84(40.8)$ \\
\hline \multicolumn{5}{|l|}{ Drug or toxin types, no. (\%) } \\
\hline Opioid analgesics and sedatives & $24(15.0)$ & $9(22.0)$ & $2(40.0)$ & $35(17.0)$ \\
\hline Other prescription medications & $38(23.8)$ & $6(14.6)$ & $1(20.0)$ & $45(21.8)$ \\
\hline Nonprescription drugs and other toxic substances & $57(35.6)$ & $15(32.6)$ & - & $72(35.0)$ \\
\hline Multiple drugs or toxic substances identified & $41(25.6)$ & $11(26.8)$ & $2(40.0)$ & $54(26.2)$ \\
\hline \multicolumn{5}{|l|}{ Level of medical training of caller, no. (\%) } \\
\hline Physician & $111(69.4)$ & $30(73.2)$ & $3(60.0)$ & $144(69.6)$ \\
\hline Registered nurse & $41(25.6)$ & $9(22.0)$ & $2(40.0)$ & $52(25.2)$ \\
\hline Other healthcare provider & $4(2.5)$ & $1(2.4)$ & - & $5(2.4)$ \\
\hline Nonhealthcare provider & $4(2.5)$ & $1(2.4)$ & - & $5(2.4)$ \\
\hline Time elapsed from initial RPCC call to death, mean \pm SD & $25.4 \mathrm{~h} \pm 67.1 \mathrm{~h}$ & $27.6 \mathrm{~h} \pm 57.3 \mathrm{~h}$ & $14.5 \mathrm{~h} \pm 18.2 \mathrm{~h}$ & $25.5 \mathrm{~h} \pm 64.3 \mathrm{~h}$ \\
\hline
\end{tabular}

$S D$ standard deviation

It is unlikely that time available for history-taking played a significant role as the mean time elapsed between the initial call and death of the victim was essentially the same. The interval for concordant and discordant cases was $25.4 \mathrm{~h}$ and $27.6 \mathrm{~h}$, respectively. The fact that, on average, providers (both hospital treating staff and RPCC staff) had 25-27 $\mathrm{h}$ to ascertain the substances involved makes it unlikely that history-taking was inhibited by time. It might be informative to study, however, what prompted the call to the RPCC in the first place and at what point in the treatment course the call was made. It has been shown that patients who receive prompt RPCC assistance have measurable reductions in average hospital lengths of stay [7]. Although the elapsed time until death is approximately 1 day, which might seem like ample time to investigate the substances involved, if RPCC consultation is delayed until the patient is in the midst of a downhill course, the treatment options become severely limited and patient prognosis may become worse. It should also be considered that discordance may be related to the difficulty in identifying many of these products and the fact that the postmortem specimens may have been obtained well after the victim's body cleared the substances but had been fatally afflicted before the substance vanished from the lab's ability to identify it.

In many instances, the initial caller had spent the longest amount of time with the patient and likely had the most detailed initial history available. An analysis of their level of training found that among the cases in which a physician contacted the RPCC, $22.9 \%$ showed discordance, while other and nonhealthcare providers demonstrated discordance in
$20 \%$ of cases. The numbers are too small to do a statistically meaningful analysis and the finding is complicated by the fact that the initial caller may not have been the primary history taker, but the trend is interesting.

It is unclear if the outcome in cases of discordance would have been any different with concordance. In several cases, although the substances reported to the poison control center were discordant with the substances found during postmortem examination, the clinical effects and treatment given may have been identical. The implications of discordance may also affect the way in which medical examiners determine poisoning as the cause of death, placing more emphasis on analysis of the clinical course of the patient. It has been reported that medical examiners and medical toxicologists may disagree on as many as $34 \%$ of cases in which poisoning was suspected as the cause of death [8]. It has also been suggested that collaboration between the RPCC and the medical examiners office may enhance the accuracy of identifying poisoning related fatalities, presumably by providing a more in-depth analysis of the clinical course of the patient [9].

The retrospective nature of this study and use of RPCC records present several limitations to our analysis. In each case, information was collected and recorded by SPIs via telephone. Although the data was collected for patient care purposes at the time of first contact and not a research study, SPIs attempted to determine all substances involved during the initial call. The key demographic and substance information were mandatory data fields in the RPCC medical record. While substances were excluded from the dataset if given 
therapeutically in the patient's course, it is possible that not all therapies were recorded in the patient's record. It has been shown, however, that completeness and accuracy of poison center data fields is relatively strong $[10,11]$. The small number of cases for which we had medical examiner toxicology results limits our ability to identify strong statistical associations in our data and precluded a more detailed analysis of the potential relationship between age, sex, location, toxic substances identified, medical qualifications of the caller, and trend over time.

While the core panel of substances tested by the medical examiner postmortem has changed somewhat during the 20 -year period our study covered, many of the substances involved such as the tricyclic antidepressants and benzodiazepines were included in the panel since 1986. Furthermore, substances such as zolpidem were not yet in production in 1986 and thus would not have been involved in a toxic ingestion. The fact that there were no significant trends over time noted in either the total number of cases or the number of discordant cases over the course of the study period supports the assumption that changes in the core panel or their sensitivities, over the study period, had little to no effect on our analysis. It is also important to remember that the postmortem examination may have been performed an appreciable time after the patient presented to the hospital and therapeutic interventions may have played a role in obscuring the toxicological evaluation.

It should be noted that because a substance is detected in postmortem analysis does not imply a causal relationship with the presenting clinical picture or the death of the patient. However, because the patient's condition prompted a call to the RPCC, there must have been some strong suspicion on the part of the treating professional that a toxic substance was involved. Although our study did not examine premortem laboratory results, toxic levels on these tests may have prompted the initial call to the RPCC.

\section{Conclusion}

It is generally accepted that poison control center consultation enhances management of the poisoned patient. Despite this, there exists an appreciable percentage $(19.9 \%)$ of cases in which there is a major difference between the substance thought to be responsible for a patients clinical course as reported to a poison center and that found on postmortem evaluation. The reasons for this discordance are currently unknown but may include a lack of thorough history-taking or a cognitive bias to the substances initially reported. It remains to be shown if the outcome in cases of discordance would have been any different with concordance. Additional studies will be needed to determine the implications of such discordance.

We suggest that prehospital and immediate hospital treating teams attempt to collect pretreatment blood on all poisoning victims and "embargo" such bloods until either the patient is discharged from the hospital or the blood is provided to the medical examiner for toxicological evaluation. Further, although our study did not reveal any evidence that discordance impacted on quality of care, the potential does exist. A close working relationship between regional poison centers providing opportunity for review of fatal cases with feedback provided to the treating physicians might be useful in promoting an improved quality of care in poisoned patients.

Conflict of Interest The authors report no conflicts of interest.

\section{References}

1. Bronstein AC, Spyker DA, Cantilena LR Jr et al (2008) 2007 Annual report of the american association of poison control centers' national poison data system (NPDS): 25th annual report. Clin Toxicol (Phila) 46:927-1057

2. Centers for Disease Control and Prevention NCfIPaC. Web-based injury statistics query and reporting system (WISQARS). 2008.

3. Finkelstein E, Corso P, Miller T (2006) The incidence and economic costs of injury in the United States. Oxford University Press, New York

4. Linakis JG, Frederick KA (1993) Poisoning deaths not reported to the regional poison control center. Ann Emerg Med 22:1822-1828

5. Croskerry P (2000) The cognitive imperative: thinking about how we think. Acad Emerg Med 7:1223-1231

6. Kovacs G, Croskerry P (1999) Clinical decision making: an emergency medicine perspective. Acad Emerg Med 6:947952

7. Vassilev ZP, Marcus SM (2007) The impact of a poison control center on the length of hospital stay for patients with poisoning. J Toxicol Environ Health A 70:107-110

8. Manini AF, Nelson LS, Olsen D, Vlahov D, Hoffman RS (2011) Medical examiner and medical toxicologist agreement on cause of death. Forensic Sci Int 206:71-76

9. Seifert SA, Letsky MC, Farooqi MF, Benson BE, Zumwalt RE (2009) A novel collaboration between a regional poison control center and state medical examiner. Clin Toxicol 47:704-705

10. Jaramillo JE, Marchbanks B, Willis B, Forrester MB (2010) Evaluation of completeness of selected poison control center data fields. J Med Syst 34:499-507

11. Lubbert J, McVoy J, Seifert SA, Jacobitz K (2005) Accuracy and completeness of initial substance identification: is what you hear, what they got. Clin Toxicol (Phila) 43:764 